\title{
Avant-propos. Normes, représentations et enjeux de la naissance dans l'Occident médiéval latin
}

\section{Carole Avignon}

\section{(2) OpenEdition}

1 Journals

Édition électronique

URL : http://journals.openedition.org/questes/735

DOI : $10.4000 /$ questes.735

ISSN : 2109-9472

Éditeur

Les Amis de Questes

\section{Édition imprimée}

Date de publication : 15 janvier 2014

Pagination : $3-11$

ISSN : 2102-7188

\section{Référence électronique}

Carole Avignon, « Avant-propos. Normes, représentations et enjeux de la naissance dans l'Occident médiéval latin », Questes [En ligne], 27 | 2014, mis en ligne le 15 janvier 2014, consulté le 25 septembre 2020. URL : http://journals.openedition.org/questes/735 ; DOI : https://doi.org/10.4000/questes.735 


\title{
Avant-propos \\ Normes, représentations et enjeux de la naissance dans l'Occident médiéval latin
}

\section{Carole Avignon}

\author{
Maître de conférences en histoire du Moyen Âge à l'Université d'Angers \\ CERHIO - Angers ; UMR 6258 CUE LUNAM
}

Comment, en quelques pages, replacer dans une perspective historique l'événement universellement partagé qui consiste à naître et en proposer une analyse qui rende compte de la multiplicité de ses implications éthiques et anthropologiques, juridiques et sociales, à l'aune des traditions culturelles de l'Occident médiéval latin? On sait bien que celles-ci procèdent des savoirs médicaux hérités de l'Antiquité grecque, mais aussi des conceptions chrétiennes de la vie, héritières de saint Augustin et de saint Jérôme ${ }^{1}$ et redevables des conceptions aristotéliciennes de l'articulation entre le corps et l'âme et du binôme forme/matière, ou encore de représentations populaires fantasmées, qui, combinés, biaisent l'appréhension des phénomènes physiologiques à l'œuvre, «de la conception à la naissance, de la grossesse à l'accouchement ${ }^{2} »$.

La naissance, entendue comme venue et mise au monde, n'est presque jamais décrite pour elle-même dans les chroniques ou les textes

\footnotetext{
${ }^{1}$ Anne Lefebvre-Teillard, «Infans conceptus. Existence physique et existence juridique ", Autour de l'enfant. Du droit canonique et romain médiéval au Code civil de 1804, Leiden, Brill, 2008, p. 53-86, en particulier p. 58-61.

2 Sylvie Laurent, Naître au Moyen Âge. De la conception à la naissance, de la grossesse à l'accouchement (XII $-X V^{e}$ siècles) [1989], Paris, Le Léopard d'Or, 1996.
} 
narratifs, et d'autant moins qu'elle est pensée comme relevant de cette intimité des femmes pour ainsi dire inconnaissable aux hommes qui produisent l'essentiel de la documentation à la disposition du médiéviste ${ }^{3}$. Parlant aussi de la «menace des sages-femmes », Myriam Greilsammer rappelle combien les représentations médiévales de naissances, notamment celle d'Anne accouchant de Marie, sont l'occasion pour les artistes (enlumineurs, sculpteurs, peintres) d'évoquer un «bastion de la solidarité, de la communion et de la toute-puissance féminines ${ }^{4} »$. Sur le ton de la satire, la «tierce joye » de mariage met en scène à la fin du Moyen Âge la jeune accouchée, entourée de ses commères, dont les exigences (culinaires) sont de plus en plus insupportables au mari épuisé de courir en tout sens pour répondre aux attentes de ces dames ${ }^{5}$. Quand dans sa Vita sua composée au début du XII ${ }^{\mathrm{e}}$ siècle, Guibert de Nogent rappelle quelles « funestes angoisses» assaillirent «son père, ses amis et ses parents» quand sa mère commença à être «bouleversée » par d'« interminables tortures », ce n'est pas pour lui l'occasion de décrire le travail de la parturiente mais bien de rappeler des peurs récurrentes devant les mystères et les dangers de l'enfantement. Au-delà de la sanction du péché originel promise à Ève (« Je multiplierai les peines de tes grossesses et dans la peine, tu enfanteras des fils $\left.{ }^{6} »\right)$, les périls de l'accouchement font bien office de «sérum de vérité » en justice pour les futures mères puisque les

${ }^{3}$ Myriam Greilsammer, L'envers du tableau. Mariage et maternité en Flandre médiévale, Paris, Armand Colin, 1990, p. 210-211. "L'enfantement n'intéresse pas. [...] Ce n'est pas l'accouchement que l'on décrit, mais les circonstances extraordinaires qui entourent une naissance dans la très haute noblesse $»$.

${ }^{4}$ Ibid., p. 223.

${ }^{5}$ Les .XV. joies de mariage, édition critique par Jean Rychner, Genève, Droz, 1990, p. 18-26.

${ }^{6}$ Genèse $3,16$. 
juges médiévaux ont accordé la même valeur au serment in doloribus partus qu'à celui prononcé à l'article de la mort? ${ }^{7}$.

Questionner l'acte de naître invite donc bien sûr le chercheur à étudier les différents acteurs en présence (parturiente, sages-femmes qu'on appelle encore matrones, nouveau-né), mais aussi à replacer l'événement, ses corollaires (en amont, la sexualité du couple conjugal, en aval, l'idée de souillure qui justifie des rituels de purification et de réintégration dans la communauté $^{8}$ ) et ses prolongements matériels et spirituels (perpétuation d'un lignage, génération d'un peuple de Dieu) dans une perspective juridique, culturelle et anthropologique spécifique.

Questionner la naissance, c'est aussi prendre en considération les représentations médiévales du corps, de l'âme et du salut, les conceptions théologiques et juridiques de la personne humaine pour qui l'esse naturale préexiste à l'esse civile et à qui l'on reconnaît une personnalité juridique (et la capacité subséquente à hériter) dès les premiers cris mais aussi un droit à renaître dans le baptême quand le fœus s'est détaché de la matrice maternelle. Se combinent la vision aristotélicienne de l'infusion de l'âme au fœetus formé (40 jours après la conception pour un garçon, 90 pour une fille ${ }^{9}$ ) et la théologie baptismale de saint Augustin qui subordonne à la naissance la capacité de recevoir la grâce du baptême puisque «celui qui n'est pas né, ne peut renaître ${ }^{10}$ » à la vie éternelle. Les traditions juridiques du droit romain et philosophiques du stoïcisme qui font commencer la vie à la naissance sont réinterprétées par le Christianisme qui étaie les

\footnotetext{
${ }^{7}$ Véronique Demars-Sion, Femmes séduites et abandonnées au XVIII siècle. L'exemple $d u$ Cambraisis, Lille, Hellemmes : ESTER, coll. «L'Espace juridique », 1991, p. 9 ; p. 131.

${ }^{8}$ Myriam Greilsammer, op. cit., p. 216-222.

${ }^{9}$ Un juge distingue les actions abortives portées contre un fœus formé comme plus graves que celles accomplies contre un fœtus non formé. (Anne Lefebvre-Teillard, op. cit., p. 59)

${ }^{10}$ De peccatis et merit., II, 2743 («qui natus non fuerit, renasci non potest ») cité par Anne Lefebvre-Teillard, op. cit., p. 60.
} 
représentations dominantes du Moyen Âge. Et ce n'est pas sans incidence dans l'élaboration intellectuelle d'une conception originale de la parenté, partagée et hiérarchisée ${ }^{11}$. Cela permet de mieux signifier que l'individu est le fruit de l'engendrement paternel, de la conception maternelle mais aussi de la création divine. Car naître est le résultat d'une "génération» physiologique et corporelle mais aussi d'une «création » divine qui touche à l'âme.

Les recherches en cours des doctorants sollicités pour le présent numéro sont bel et bien justifiées par la pluralité des déclinaisons d'un tel sujet, ainsi que sa pertinence pour questionner les rapports de genre (fondamentalement articulés par le binôme charnel/spirituel), les systèmes de représentation (dans une société aristocratique fortement marquée à partir $\mathrm{du} \mathrm{XII}^{\mathrm{e}}$ siècle par l'émergence de la conscience lignagère et l'importance de la pureté du lignage garantie par la fidélité de la mèreépouse, mais aussi dans une Chrétienté médiévale attachée à l'idée de germanité des hommes, tous fils de Dieu), ou les rapports sociaux, notamment entre laïcs et clercs (ceux-ci s'étant vus confier la tâche de contrôler le mariage pour mieux contrôler la sexualité de ceux-là, éléments discriminants par essence dans l'esprit de la Réforme Grégorienne, entre ces deux états dans le plan divin de salut). Les quelques remarques liminaires que je formule ici ne sauraient donc bien sûr épuiser une telle matière.

Les renouvellements de la production scientifique sur l'existence, réelle et documentée, d'un «sentiment de l'enfance » au Moyen Âge ont produit une abondante littérature sur les soins que médecins et pédagogues recommandent aux futures mères et aux fruits qu'elles portent ainsi qu'à l'infans dont la fragilité renforce la totale dépendance à la mère ou la

${ }^{11}$ Jérôme Baschet, Le sein du père. Abraham et la paternité dans l'Occident médiéval, Paris, Gallimard, 2000, chapitre 8, p. 310-343. 
nourrice. Nutrire c'est autant alimenter qu'élever, éduquer l'enfant. L'attention portée à l'histoire des femmes n'a pas pu faire non plus l'économie d'une histoire des mères et de la maternité, tant le corps féminin est fondamentalement pensé au Moyen Âge comme un «corps maternel », tant l'«utéro-centrisme » est fort dans la pensée médicale, théologique et pastorale ${ }^{12}$. Depuis Ève, la maternité justifie la création de la femme comme être sexué, et assure son salut si la procréation, et donc la sexualité, sont bien accomplies dans le cadre légal de l'institution matrimoniale. Sexualité et féminité sont le résultat de deux concessions combinées quand on lit dans la Première Épître à Timothée $(2,15)$ de saint Paul: «Néanmoins, elle sera sauvée en devenant mère, à condition de persévérer avec modestie dans la foi, la charité et la sainteté ${ }^{13} »$. Jérôme commentant l'Épitre aux Éphésiens n'écrit-il pas à son tour : «Tant que la femme est faite pour la naissance et les enfants, elle est différente des hommes comme le corps l'est de l'âme. Mais lorsqu'elle fait vœu de servir le Christ plus que le monde, elle cessera d'être une femme et sera appelée un homme ${ }^{14} \gg$. Féminité et maternité conjugale sont bien à envisager conjointement.

Le mariage n'est pas pensé comme le cadre institutionnel exclusif pour permettre d'établir la légitimité de la filiation dans la seule tradition chrétienne puisqu'on retrouve ce même principe dans les systèmes juridiques romain et hébraïque. Mais la théologie chrétienne, héritière de saint Augustin, a poussé très loin la corrélation entre mariage et procréation. Plus qu'une finalité du couple conjugal, héritière de la Création quand Dieu prescrit à Adam et Ève, faits homme et femme, d'être

\footnotetext{
${ }^{12}$ Didier Lett, Hommes et femmes au Moyen Âge. Histoire du genre, XII $-X V^{e}$ siècles, Paris, Armand Colin, 2013, p. 32-33 : « [...] dans les traités de médecine, en dehors des chapitres relatifs à la génération et à la gynécologie, le corps féminin n'existe pas ».

${ }^{13}$ Cité par Didier Lett, op. cit., p. 33.

${ }^{14}$ Cité par Didier Lett, op. cit., p. 99.
} 
« féconds » (Genèse 1, 28), la production d'une descendance, proles est présentée comme un bien du mariage, au même titre que la fides et le sacramentum. Pour l'auteur du De nuptiis et concupiscentia, ceux qui refusent volontairement la procréation ne sont plus «époux» mais « fornicateurs ». La découverte de l'impuissance du mari permet quant à elle de considérer nul un mariage. Le désir de l'épouse de s'accomplir comme mère est presque toujours avancé dans les dépositions des femmes qui engagent une action auprès du tribunal de l'évêque (l'officialité). Si ces femmes peuvent obtenir de l'official « licence de se marier ailleurs », c'est parce que la «frigidité » du mari n'a pas permis la perfection du contrat matrimonial par la copula carnalis, mais aussi parce que ce dernier ne saurait parvenir à «facere matrem » ces femmes qui ne manquent pas de mettre en avant leur désir de «procreare filios $»^{15}$.

Pour que puisse «naître» un enfant, il faut qu'un homme l'ait engendré, qu'une femme l'ait conçu, puis enfanté. Cette approche genrée du vocabulaire de la procréation que détaille Jérôme Baschet rend compte de conceptions médiévales où savoirs médicaux et principes théologiques se conjuguent. Jusqu'au XII ${ }^{\mathrm{e}}$ siècle, dominent les conceptions galénistes qui reconnaissent que la semence de la femme (certes moins active) se combine à celle de l'homme pour former l'embryon. Mais à partir du XIII ${ }^{\mathrm{e}}$ siècle, la théorie aristotélicienne s'impose et avec elle, triomphe l'idée que seul l'homme produit un semen qui engendre tandis que la femme fournit la matrice à la conception ${ }^{16}$. Engendrement et génération sont des actions masculines, à l'image du Pater genitor de la Trinité. La supériorité séminale du père sur la mère est toutefois relativisée par l'action supérieure encore accomplie par Dieu dans l'infusion de l'âme au corps. Pierre

\footnotetext{
${ }^{15}$ Martine Charageat, Délinquance matrimoniale. Couples et justice en Aragon (XV ${ }^{e}$ $X V I^{e}$ siècle), Paris, Publications de la Sorbonne, 2011, note 72, p. 111.

${ }^{16}$ Jérôme Baschet, op. cit., p. 324-325. 
Lombard et Thomas d'Aquin adoptent le «créationnisme » reconnu par saint Jérôme en son temps, c'est-à-dire l'idée selon laquelle « chaque âme est créée lors de la conception charnelle de l'enfant et aussitôt infusée dans l'embryon », ainsi que le figure la célèbre enluminure du recueil de littérature morale destiné à Baudouin de Lannoy, à la fin $\mathrm{du} \mathrm{XV}^{\mathrm{e}}$ siècle $^{17}$. Dans sa Somme Théologique, saint Thomas précise qu' « une substance immatérielle et incorporelle ne peut être produite par la génération, mais seulement par la puissance créatrice de Dieu ${ }^{18}{ }^{1}$. Jérôme Baschet a pu démontrer aussi que le christianisme introduit également une rupture dans la succession biblique des engendrements masculins, celui d'un «engendrement maternel», hors-norme, incarné par la Vierge Marie ${ }^{19}$. Ainsi la généalogie du Christ qui ouvre le Nouveau Testament prend-elle une dimension nouvelle quand on lit : Jacob genuit Joseph, virum Mariae de qua natus est Jesus (Matthieu 1). Cet engendrement maternel est en fait celui de l'Église « qui engendre les Chrétiens dans le baptême, tout comme Marie engendre virginalement le $\mathrm{Christ}^{20}{ }^{2}$.

Naître, ne l'oublions pas, est aussi un événement privé à fortes résonances économiques et sociales, de ceux qu'on voit consignés par certains chefs de famille dans les documents du for privé que sont les ricordanzi ou les livres de raison. La naissance d'un enfant constitue alors la promesse d'un héritier, ou suscite l'appréhension devant l'arrivée d'une nouvelle bouche à nourrir. À partir du $\mathrm{XVI}^{\mathrm{e}}$ siècle, les chroniqueurs

\footnotetext{
${ }^{17}$ Ibid., p. $327-328$.

${ }^{18}$ Ibid., p. 328.

${ }^{19}$ Le vocabulaire graphique des scènes de naissance présentées dans la célèbre Bible de Pampelune achevée en 1197 témoigne de la posture dominante du père quand la mère est significativement présentée alitée et passive (naissance d'Isaac, notamment). Ibid., p. 319. L'interprétation de ces scènes n'est en rien contradictoire avec celles, plus intimistes et exclusivement féminines, présentées notamment dans le travail de Myriam Greilsammer, ou dans L'Enfance au Moyen Âge (Pierre Riché, Danielle Alexandre-Bidon, Paris, Seuil, Bibliothèque Nationale de France, 1994, notamment p. 46.)

${ }^{20}$ Jérôme Baschet, op. cit., p. 323.
} 
flamands étudiés par Myriam Greilsammer commencent à donner d'intéressants détails sur la composition et l'évolution de leur famille: ainsi consignent-ils la date (jour, mois, année) de naissance des enfants, faisant parfois exception toutefois de ceux qui sont morts en bas âge, rappel de la forte mortalité infantile. Louis Porquin, financier d'origine italienne marié à une Brugeoise, précise bien qu'il entend ainsi laisser à ses enfants « ce qui est le plus important pour [eux], c'est-à-dire le temps exact de chacune des naissances en particulier, et le nom de [leurs] parrains et marraines, ce qui [leur] laissera dans le cœur une mémoire particulière de [leurs] parents ${ }^{21} »$. Au Moyen Âge, l'état civil n'existe pas et seule la mémoire familiale et communautaire peut y suppléer. Les premiers registres dont la tenue est prescrite par l'Église ne furent d'ailleurs pas des registres de naissances, mais des registres de baptême (de manière à pouvoir identifier ceux des parents spirituels qui ne sont pas éligibles au mariage). Cela invite aussi subsidiairement à reposer la question de savoir ce qui fonde l'intégration à la communauté : la naissance physique ou la renaissance spirituelle dans le baptême? Anne Lefebvre-Teillard a bien montré que la première permettait d'établir le point de départ de la personnalité juridique, - notamment pour être considéré comme capable d'hériter, sauf cas particulier de l'enfant posthume pour lequel fut reçue dans la théorie juridique médiévale la maxime du Digeste «infans conceptus pro nato habetur quoties de commodis ejus agitur» (Digeste $1,5,7)$. Certaines lois germaniques allèrent au-delà en subordonnant l'attribution de la personnalité juridique à l'attribution du nom, elle-même effective quand la capacité à survivre de l'enfant semble établie. Ce principe survécut dans l'attribution coutumière de capacités successorales au nouveau-né s'il est établi qu'il a pleuré à la naissance (signe de sa viabilité). Philippe de Beaumanoir reprend à son compte ces conditions

${ }^{21}$ Cité par Myriam Greilsammer, op. cit., p. 213. 
mais récuse celle qui attacherait la personnalité juridique du nouveau-né à son baptême ${ }^{22}$. Des coutumes médiévales espagnoles, valaisannes ou françaises continuent toutefois de subordonner la capacité d'hériter de l'enfant posthume à son baptême. Renaissance en Dieu, le baptême est aussi une cérémonie publique qui fait entrer l'individu dans la communauté, comme en témoigne par exemple la coutume de réserver l'attribution d'un nom à l'enfant lors des rituels de baptême pratiqués à l'église même (et exclut donc l'attribution d'un nom par les laïcs contraints d'ondoyer en urgence le nouveau-né dont la vie et le salut sont en péril $)^{23}$.

« Naissances »: l'objet de recherche engagée dans les pages qui vont suivre mérite donc autant un pluriel qu'une minutieuse mise en perspective culturelle, juridique et sociale.

${ }^{22}$ Anne Lefebvre-Teillard, op. cit., passim.

${ }^{23}$ Telle est du moins la discipline prescrite dans les statuts synodaux en usage dans l'Ouest de la France, dans la tradition du synodal de l'Ouest. Anne LefebvreTeillard, "Baptême et nom de baptême. Notes sur l'ondoiement», Autour de l'enfant. Du droit canonique et romain médiéval au Code Civil de 1804, Leiden, Brill, 2008, p. 87-94. 